\title{
Research on Electro-hydraulic Control System of the Special Section
}

\author{
Qiu-jun Huang ${ }^{\mathrm{a} 1}$, Jin-chun Song ${ }^{\mathrm{b2,},}$, Yan Cai $^{\mathrm{c3}}$ and Tian-zhong Huang ${ }^{\mathrm{d} 4}$ \\ 1, 2, 3, 4 School of Mechanical Engineering and Automation, Northeastern University, Shenyang, \\ Liaoning, China \\ bjchsong@mail.neu.edu.cn \\ ${ }^{*}$ Corresponding author
}

Keywords: Electro-hydraulic Control, Special Section, Shield Tunneling Machine.

\begin{abstract}
Shield tunneling machine is a kind of large-scale high-tech construction equipment specialized for the underground tunneling. Traditional shield tunneling machine with the circular-section has problems such as low utilization ratio of cross-section, waste of space and so on. In this paper, we present a new mechanism of the special-shape cross-section shield cutting mechanism, which can reduce the excavation area, reduce the amount of backfill soil, thus improving the efficiency and space utilization. We establish the calculation model of a section of cutting mechanism and analysis the motion requirements of the special section shield cutting mechanism, design the electro-hydraulic proportional control system. We establish the mathematical model of asymmetric hydraulic cylinder controlled by asymmetric proportional directional valve, obtain the transfer function of the electro-hydraulic proportional position control system of the cutting mechanism, and the simulating model of the hydraulic is built based on MATLAB/Simulink. The application of PID in control system can effectively solve the instable problem of system, obtain better system response and provide a theoretical basis for later analysis.
\end{abstract}

\section{Introduction}

Shield tunneling machine is a kind of large-scale high-tech tunnel construction equipment which is specialized used in underground tunnel project [1]. It has the advantages of fast excavation, high quality, safety, economy, protecting environmental and reducing labor intensity. The main cross section of traditional shield machine is circular-section [2]. However, it is found that there are many disadvantages when digging special-section tunnel with circular-section shield machine, such as low efficiency, space wasted, etc. With the development and utilization of the underground tunneling, we have higher demands for the shield tunneling machine [3]. Digging special-section tunnel with special-section shield machine can reduce digging area and the amount of backfill soil, thus improving space utilization, efficiency and making the tunnel construction technology more advance. Therefore, the tunneling shield machine with variously special-shape cross-section is need in the future [4]. So the most urgent problem for researching and resolving is design and development the structure of special section shield machine. First we have to solve the main part of shield tunneling machine that cutter cutting mechanism.

\section{Design Special-shape Cross-section Cutting Mechanism}

To solve the existed technology problem of traditional shield tunneling machine, we design a special-shape cross-section cutting mechanism for shield tunneling machine. The cutting mechanism breaks through the technological bottlenecks that the existing shield tunneling machine without various section cross. The diagram of special-shape cross-section cutting mechanism is shown in fig.

\section{Working Principle}

The driving mechanism of the cutter disk is made up of the driving motor and reducer components. Mechanism drives the cutter disk rotation. The swing cylinder drives the support arm swing. The 
telescopic cylinder drives the cutter rectilinear motion along the axis of the telescopic arm. Therefore, the cutter can draw a given curve on the cross-section and obtain various special-shape cross-section.

The path curve is accurately controlled by the close-loop control system. First, analysis the geometrical relationship between the special-shape of tunneling cross-section and shield cutting mechanism. Second, obtain the function of the swing arm, telescopic arm and the cutter. Third, compile the function in the controller. Final, according the feedback data from the sensor, the control system can accuracy adjust position of the telescopic cylinder and swing cylinder, to make sure the path of cutter is correct.

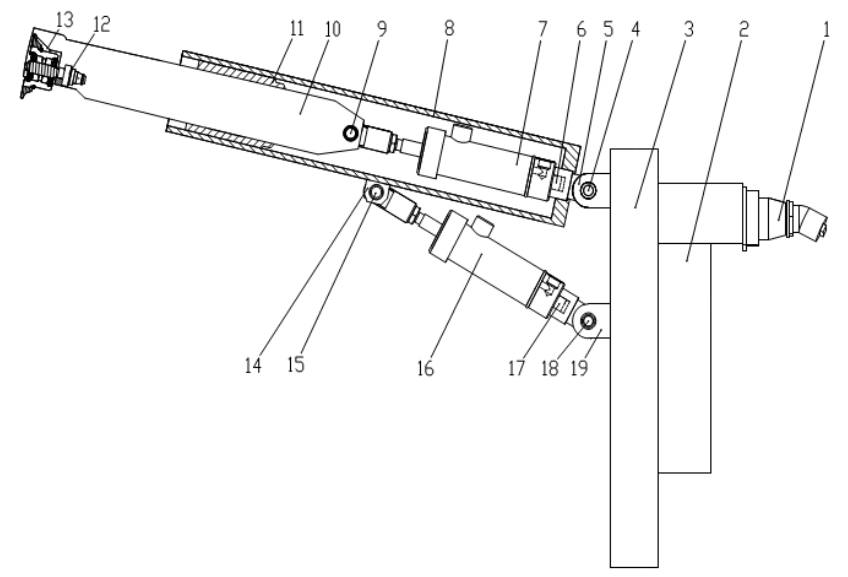

1. Driving motor; 2. Reducer components; 3. Cutter disk; 4,9,15. Pin; 5, 14, 19. Earrings;6, 17. Displacement sensor; 7.Telescopic cylinder; 8. Telescopic arm; 10. Cutting arm;11. Guide sleeve; 12. Cutting motor; 13. Cutter; 16. Swing cylinder

Figure 1. Diagram of special-shape cross-section shield cutting mechanism

\section{Calculation Model}

For example, using the special-shape shield mechanism tunnels a doorway-shape cross-section. We should establish and theoretically calculate the calculation model of cutting mechanism of doorway-shape cross-section.

Point $\mathrm{B}$ is the position of cutter of special-shape shield mechanism. Point $\mathrm{P}$ is the starting point of the cutter. Angle $\theta$ is the include angle of $\mathrm{O}^{\prime} \mathrm{B}$ and $\mathrm{O}^{\prime} \mathrm{P}$. Line $\mathrm{R}$ is the radius of the circular arc on the doorway-shape. The height of the doorway-shape is $\mathrm{m}$. Divide the path curve of doorway-shape into four segments, the functions of the path can be obtained as:

$$
\mathrm{O}^{\prime} \mathrm{B}=\left\{\begin{array}{l}
R, \quad \theta \in(0, \pi] \\
\frac{R}{-\cos \theta}, \theta \in\left(\pi, \pi+\arctan \frac{m}{R}\right] \\
\frac{m}{-\sin \theta}, \theta \in\left(\pi+\arctan \frac{m}{R}, 2 \pi-\arctan \frac{m}{R}\right] \\
\frac{R}{\cos \theta}, \theta \in\left(2 \pi-\arctan \frac{m}{R}, 2 \pi\right]
\end{array}\right.
$$

The diagram of cutting mechanism tunneling a doorway-shape cross-section is shown in fig.3. The disk in the right is cutter disk. Line $\mathrm{BC}$ represents telescopic arm. We give line AD representation for swing arm. Point $\mathrm{A}$ is the hinge joint of telescopic arm and swing arm. Point $\mathrm{C}$ represents the hinge joint of the telescopic arm and the cutter disk. Point $\mathrm{D}$ represents the hinge joint of the swing arm and the cutter disk. Line O'O represents the horizontal distance of cutter disk and cross-section. 
The geometric relationship of cutting mechanism tunneling cross-section is shown in fig 4. Set $\mathrm{O}^{\prime} \mathrm{B}=1$, in the right triangle $\Delta \mathrm{OO}^{\prime} \mathrm{B}$, by the Pythagorean Theorem

$$
\mathrm{BO}^{2}=\mathrm{O}^{\prime} \mathrm{O}^{2}+\mathrm{BO}^{\prime 2}=\mathrm{l}^{2}+\mathrm{R}^{2}
$$

Set $\angle \mathrm{OCB}=\varphi$, in the right triangle $\triangle \mathrm{OO}$ 'B, by the Cosine Theorem:

$$
\cos \varphi=\frac{\mathrm{OC}^{2}+\mathrm{BC}^{2}-\mathrm{OB}^{2}}{2 \mathrm{OC} \times \mathrm{BC}}
$$

In the triangle CDA, by the Cosine Theorem:

$$
\cos \varphi=\frac{\mathrm{CD}^{2}+\mathrm{CA}^{2}-\mathrm{AD}^{2}}{2 \times \mathrm{CD} \times \mathrm{CA}}
$$

In the right trapezoid $\mathrm{OO}^{\prime} \mathrm{CB}$, set $\angle \mathrm{OCB}=\varphi, \angle \mathrm{ECB}=\beta$, the equation can be written as:

$$
\cos \varphi=\cos \left(\frac{\pi}{2}+\beta\right)=-\sin \beta=-\frac{\mathrm{O}^{\prime} \mathrm{B}-\mathrm{OC}}{\sqrt{\left(\mathrm{O}^{\prime} \mathrm{B}-\mathrm{OC}\right)^{2}+1^{2}}}
$$

Combine equation (1)-(5) given above, the length of swing arm AD and the length of telescopic arm BC can be calculated.

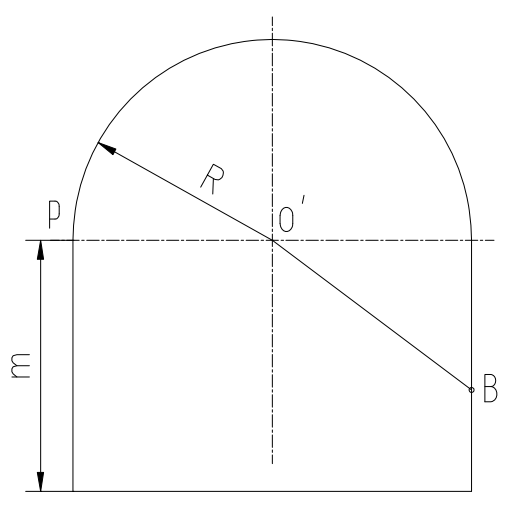

Figure 2. The cross-section of doorway-shape

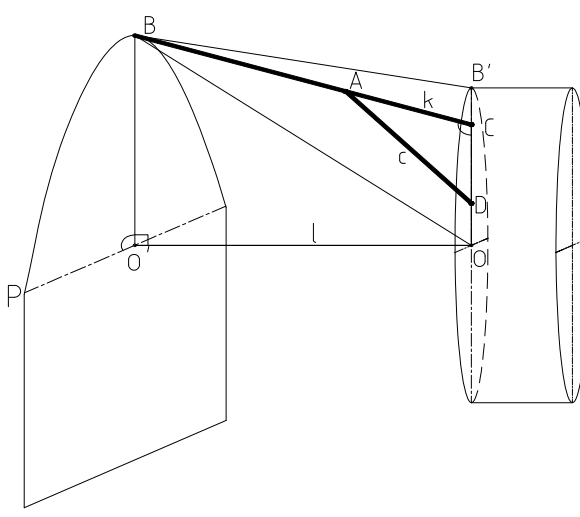

Figure 3. The diagram of cutting mechanism tunneling cross-section

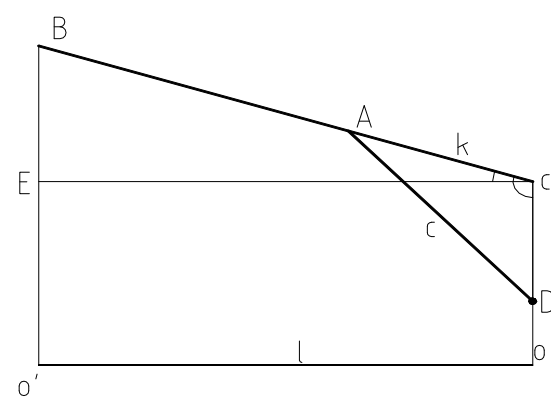

Figure 4. The geometric relationship of cutting mechanism tunneling section

\section{Hydraulic Control System of Cutting Mechanism}

The electro-hydraulic control system of cutting mechanism includes following control processes:

(1). Quantify the curve path on special-shape tunneling cross-section and establish its mathematical model.

(2). Write the control program into computer according the mathematic model.

(3). Sensors on the swing cylinder, telescopic cylinder and cutter disk can simultaneously record the dynamic displacement of the piston rod and the cutter disk.

(4). A/D converter converts the data to the digital signal and transfers the signal to the computer.

(5). Control system in computer analyzes the input digital signal and output the control signal to control the hydraulic proportional valves motions.

(6). Electro-hydraulic close-loop control system achieves accurately controls the motion path of cutter. 
As shown in fig. 5, the scheme of electro-hydraulic proportional control system is decided. Using the electro-hydraulic close-loop control system, the cutting mechanism can obtain the cross-section that we need. The hydraulic system's actuator consists of two cylinders and hydraulic motor. Fig. 5 shows the control principle of the electro-hydraulic proportional system of tunnel mechanism. Because the need oil pressure in hydraulic circuit of cylinders and hydraulic motor are different, system set a pressure reducing valves on the hydraulic circuit of cylinders. The proportional valves can control the flow and pressure of cylinders. The direction and speed of rotation of the hydraulic motor are controlled by electromagnetic valve and the throttle valves.

\section{The Analysis of Hydraulic Control System}

The shield cutting mechanism consists of control and measurement, cutter and cutter disk, electro-hydraulic control three key technology. It is greatly important that analysis the hydraulic system of cutting mechanism [5]. The valve-controlled mode are applied to control the hydraulics cylinder and motor of this system. Following with modeling for the hydraulic control system with transfer function.

\section{Proportional Directional Valve Model}

According the structure and working principle of the proportional valve, it can be regard as a second order [6]. The transfer function of proportional valve can be written as:

$$
W_{p v}(s)=\frac{K_{q}}{\frac{s^{2}}{\omega_{v}^{2}}+\frac{2 \delta_{v} s}{\omega_{v}}+1}
$$

Where $\mathrm{Kq}$ is the flow gain of proportional directional valve, ${ }^{\omega_{v}}$ represents the phase bandwidth, $\delta_{v}$ is the damping ratio of proportional.

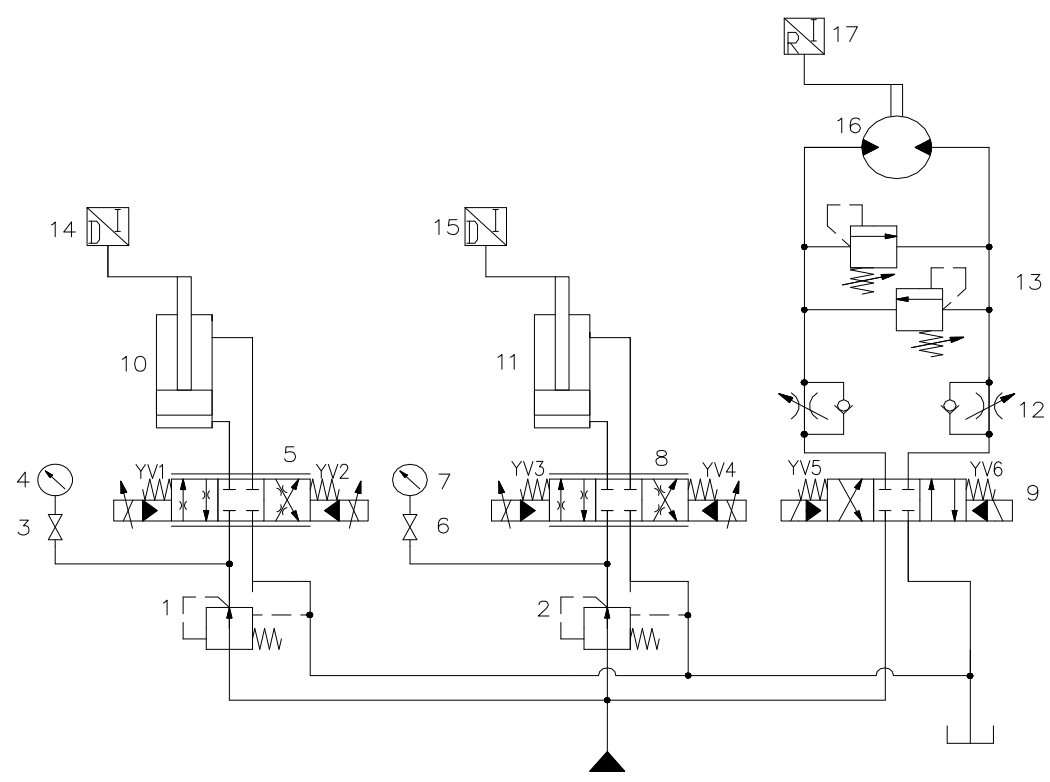

1, 2 - Pressure reducing valve; 3, 6-Globe valve; 4, 7 - Pressure gauge; 5, 8 - Three-position four-way proportional directional valve; 9 -Three-position four-way electromagnetic directional valve; 10 - Swing cylinder;11 - Telescopic cylinder; 12 - Throttle valve; 13 - Relief valve;14, 15 - Displacement sensor; 16 - Hydraulic motor; 17 - Angle sensor

Figure 5. Schematic diagram of the hydraulic system of special-shape tunneling mechanism 


\section{Proportional Amplifier Model}

The corner frequency of proportional amplifier is much higher than bandwidth [7], so the proportional amplifier is regarded as proportional order, it can be written as:

$$
K_{a}=\frac{I_{0}(s)}{I_{i}(s)}
$$

Where $K_{a}$ is gain of proportional amplifier, $I_{0}(s)$ and $I_{i}(s)$ each represents the output and input current of proportional amplifier.

\section{Displacement Sensor Model}

The bandwidth of displacement sensor is much higher than bandwidth of system, so the displacement sensor can be regarded as proportional order, it can be written as:

$$
K_{\mathrm{m}}=\frac{I(s)_{\max }}{x(s)_{\max }}
$$

Where $K_{\mathrm{m}}$ is the gain of proportional gain.

\section{Asymmetrical Valve Controlled Asymmetrical Hydraulic Cylinder Model}

The control model of valve-control cylinder system of the cutting mechanism can be chosen by the actual working condition. In this paper, we choice the asymmetrical valve control asymmetrical cylinder, the transfer function of the simplified model can be written as:

$$
X_{\mathrm{p}}=\frac{\frac{K_{q}}{A_{p}} X_{v}-\frac{1}{A_{N} A_{p}}\left(K_{\mathrm{ce}}+\frac{V_{e}}{4 \beta_{\mathrm{e}}} s\right) F_{\mathrm{L}}}{\frac{V_{e} m_{\mathrm{t}}}{4 \beta_{\mathrm{e}} A_{N} A_{p}} s^{3}+\frac{K_{\mathrm{ce}} m_{\mathrm{t}}}{A_{N} A_{p}} s^{2} s}
$$

where ${ }^{A_{N}}$ is the effective area of the cylinder, ${ }^{X_{\mathrm{v}}}$ is the displacement of proportional valve element, when $X_{v}>0, \mathrm{~N}=1$, when $X_{\mathrm{v}}<0, \mathrm{~N}=2, K_{q}$ is the flow gain, $K_{\text {ce }}$ is the equivalent pressure-low coefficient.

\section{Diagram of Hydraulic Control System}

The hydraulic natural frequency represents the response speed of hydraulic power component. In the hydraulic proportional control system, the hydraulic natural frequency is the lowest frequency during the whole system, so it limits the response speed of system. The block diagram of proportional control system is constructed in fig. 6 .

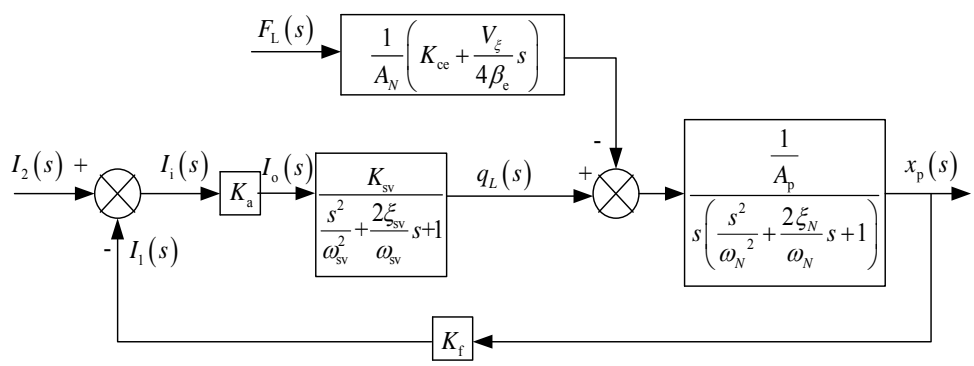

Figure 6. Block diagram of the hydraulic proportional control system 
The system simulation model is established on Matlab/Simulink software, the simulation model block diagram is constructed in fig. 7.

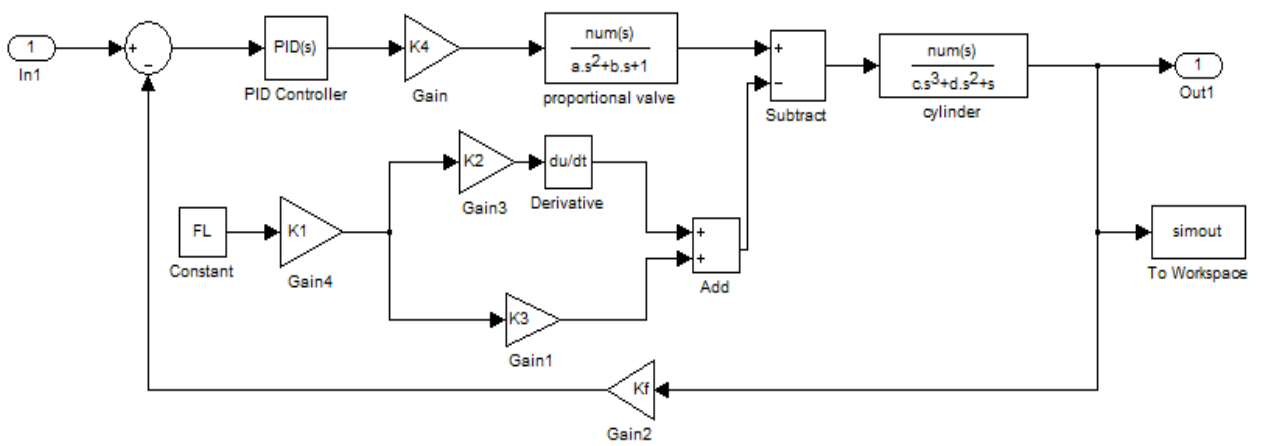

Figure 7. Simulation block diagram of the proportional control system

\section{Performance Analysis of Hydraulic System}

Take the parameters into the simulation model block diagram in fig. 7, the system step response curve when the rod retraction can be obtained as fig. 9. The step response curves in fig. 8 shows that the response time of rod retraction $t_{s}=0.743 \mathrm{~s}$. The step response curve with PID controller system is shown as the fig. 9. The figure shows that the response time of rod retraction $t_{s}=0.106 \mathrm{~s}$. The response speed can meet the demands of the system requirement, after using PID controller. It can be concluded from the simulation results that adjusting the PID parameters can obtain a quicker and stable response control system to meet the demand of the control system.

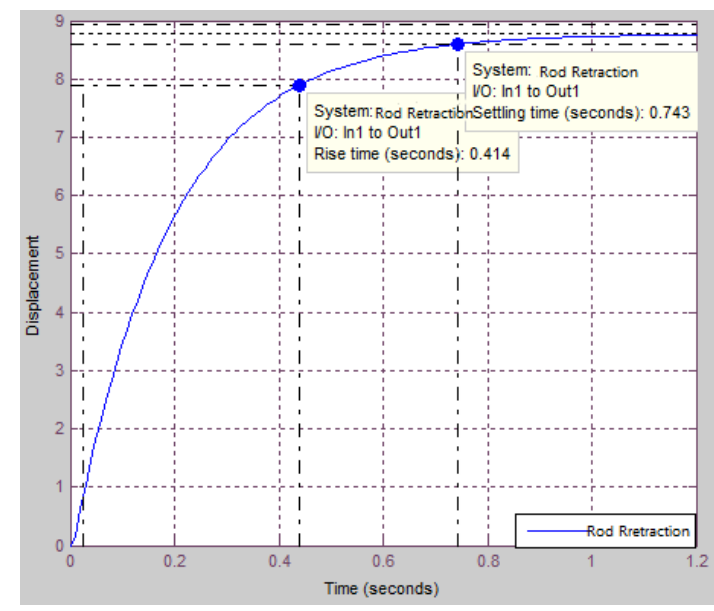

Figure 8. Step response curve when the rod retraction

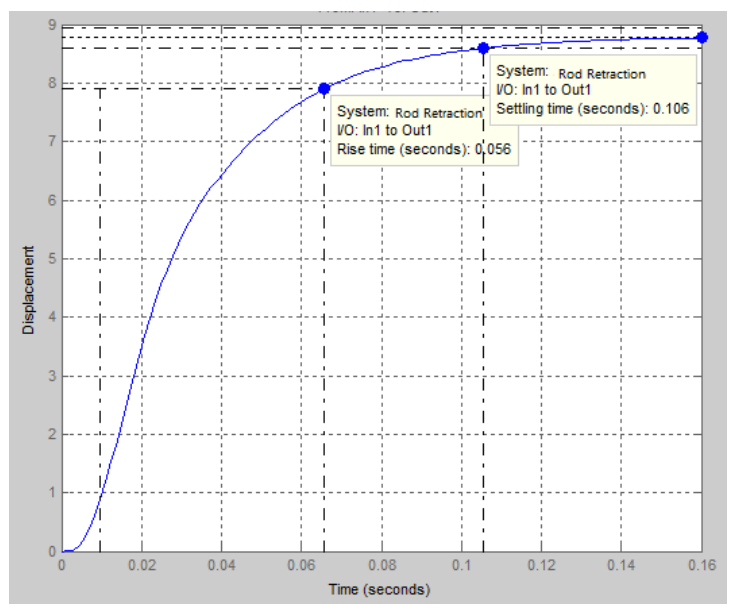

Figure 9. Step response curve of PID control system

when the rod retracts

\section{Conclusions}

This paper proposes a new type special-shape cross-section shield tunneling mechanism which is control by electro-hydraulic proportional system. The mechanism can tunnel complex special-shape cross-section, such as doorway-shape and rectangle-shape. The cutter can tunnel the special-shape cross-section in one time. The mechanism can straightly exchange the cutter disk of traditional shield, so it is very well interchangeable. In addition, this paper has established computational model of the cutting mechanism tunneling cross-section, designed the electro-hydraulic proportional control system, obtained the transfer function of electro-hydraulic proportional displacement control of the 
mechanism and established the simulation model on Matlab/Simulink software. It can provide a theory foundation to future analysis.

\section{References}

[1] H. Shi, H. Yang, G. Gong, L. Wang, Determination of the cutter head torque for EPB shield tunneling machine. Automation in Construction 20.8 (2011): 1087-1095.

[2] Q. Zhang, C. Qu, Z. Cai, Y. Kang, T. Huang, Modeling of the thrust and torque acting on shield machines during tunneling. Automation in Construction 40 (2014): 60-67.

[3] L. Wang, G. Gong, H. Shi, H. Yang, Modeling and analysis of thrust force for EPB shield tunneling machine. Automation in construction 27 (2012): 138-146.

[4] K. Deng, X. Zhang, J. Yang, H. Wang, Deformation characteristics under variable stiffness for the propelling mechanism of EPB shield machines in mixed ground. Journal of Mechanical Science and Technology 28.9 (2014): 3679-3685.

[5] T. Z. Huang, J. C. Song, Y. Cai, Design of the Cutting Mechanism of Special Section Shield and Its Hydraulic, Machinery Design and Manufacture System, 10(2014) 79-81.

[6] K. Deng, H. Wang, Analysis of the carrying capacity of the propelling mechanism of tunneling machines. Journal of Mechanical Science and Technology 29.8 (2015): 3343-3349.

[7] Y. Huayong, S. Hu, G. Guofang, H. Guoliang, Electro-hydraulic proportional control of thrust system for shield tunneling machine. Automation in Construction 18.7 (2009): 950-956. 